\title{
Intérêt du dosage de l'activité cytochrome oxydase pour l'évaluation de la flore psychrotrophe du lait cru à l'arrivée à l'usine
}

\author{
D Rongvaux-Gaïda, C Piton-Malleret * \\ INRA, station de recherches en technologie et analyses laitières, \\ BP 89, 39801 Poligny cedex, France
}

(Reçu le 7 mars 1991; accepté le 6 décembre 1991)

\begin{abstract}
Résumé - Cent vingt échantillons de lait cru ont été prélevés dans les citernes de ramassage de 4 grandes entreprises laitières, dès leur arrivée au quai. Après agitation pendant $30 \mathrm{~s}$ à l'aide d'un Ultraturrax, les échantillons ont été soumis au dénombrement de la flore psychrotrophe à l'aide du système "Spiral". L'activité enzymatique de la cytochrome oxydase a été déterminée en double après une incubation préliminaire des échantillons : $7 \mathrm{~h}$ à $30^{\circ} \mathrm{C}$ ou $48 \mathrm{~h}$ à $4{ }^{\circ} \mathrm{C}$ ou $48 \mathrm{~h}$ à $4{ }^{\circ} \mathrm{C}$ suivies d'une incubation supplémentaire de $7 \mathrm{~h}$ à $30^{\circ} \mathrm{C}$. L'oxydation du TMPD (NNN'N' tétraméthyl-p-phénylène diamine dihydrochloride) par la cytochrome oxydase se traduit par l'apparition d'une coloration bleu-violette, dont l'intensité a été évaluée, soit visuellement par comparaison à une gamme étalon, soit à l'aide d'un chromamètre. Les intensités de coloration mesurées par les 2 modes de lecture sont très corrélées $(r=0,98)$. Les écarts types relatifs géométriques de répétabilité (en $\%$ UFC/ml) sont dans tous les cas inférieurs à $10 \%$ pour la lecture visuelle et la mesure colorimétrique. Pour les échantillons de lait analysés après une préincubation de $7 \mathrm{~h}$ à $30^{\circ} \mathrm{C}$, il n'existe pas de corrélation satisfaisante entre l'intensité de coloration et le niveau de la flore psychrotrophe initiale. Cependant, après $48 \mathrm{~h}$ d'incubation à $4^{\circ} \mathrm{C}$, il apparaît une relation linéaire sur toute la gamme de concentration des échantillons analysés (entre $10^{3}$ et $10^{8} \mathrm{UFC} / \mathrm{ml}$ ); l'écart type résiduel de la régression est de $0,524 \mathrm{log} \mathrm{UFC} / \mathrm{ml}$ dans le cas d'une lecture visuelle, contre $0,534 \mathrm{log}$ UFC/ml dans le cas d'une lecture au colorimètre. Une incubation supplémentaire de $7 \mathrm{~h}$ à $30^{\circ} \mathrm{C}$ n'améliore pas la précision d'estimation du dosage : l'écart type résiduel est alors de $0,523 \log \mathrm{UFC} / \mathrm{ml}$ dans le cas d'une lecture visuelle.
\end{abstract}

cytochrome oxydase / activité enzymatique / lecture visuelle / lecture colorimétrique / lait cru / flore psychrotrophe / répétabilité / justesse

Summary - Use of the cytochrome oxidase test for the evaluation of psychrotrophic flora in raw milk at the dairy plant. One hundred and twenty milk-tanker road samples were collected from 4 French dairy plants to evaluate the analytical performances of the cytochrome oxidase activity assay used to assess the bacteriological quality of raw milk. The samples were submitted to shaking in an Ultra-turrax for $30 \mathrm{~s}$ and then to enumeration of the psychrotrophic flora using the Spiral method. The cytochrome oxidase activity was also determined, in duplicate, after preincubation of the samples: $7 \mathrm{~h}$ at $30^{\circ} \mathrm{C}$ or $48 \mathrm{~h}$ at $4{ }^{\circ} \mathrm{C}$ or $48 \mathrm{~h}$ aे $4{ }^{\circ} \mathrm{C}$ followed by an additional incubation of $7 \mathrm{~h}$ at $30^{\circ} \mathrm{C}$. The TMPD (NNN'N'-tetramethyl-p-phenylene-diamine dihydrochloride) oxidation by cytochrome oxidase gave a blue coloration; its intensity was determined by 2 methods: visually by comparison with a scale, or with a colorimeter. Both color intensity measures were very well correlated $(r=0.98)$ and

* Adresse actuelle : INRA, laboratoire de recherches sur la viande, CRJ, 78352 Jouy-en-Josas Cedex, France 
the repeatability geometric relative standard deviations (in \% CFU/ml) were lower than $10 \%$ for both the visual and colorimeter readings. For samples analysed after a $7-h$ preincubation at $30^{\circ} \mathrm{C}$, no correlation was found between color intensity and the level of the initial psychrotrophic flora (in log CFU/ $\mathrm{ml}$ ). After a 48 - $h$ preincubation at $4^{\circ} \mathrm{C}$, a linear relationship appeared for all the bacterial concentration range of samples analysed (between $10^{3}$ and $10^{8} \mathrm{CFU} / \mathrm{ml}$ ); the residual standard deviation of the regression was $0.524 \mathrm{log}$ CFU/ml for the visual reading and $0.534 \mathrm{log}$ CFU/ml for the colorimeter reading. An additional incubation of $7 \mathrm{~h}$ at $30^{\circ} \mathrm{C}$ did not improve the accuracy of the method: the residual standard deviation of the regression was then $0.523 \mathrm{log}$ CFU/mi for visual reading.

cytochrome oxidase / enzymatic activity / visual reading / colorimetric reading / raw milk / psychrotrophic flora / repeatability / accuracy

\section{INTRODUCTION}

L'introduction de la réfrigération du lait à la ferme et l'espacement des collectes (tous les 2-3 j) ont entraîné une modification de la flore présente dans le lait cru. En effet, le lait non refroidi contenait des bactéries lactiques, dont l'activité principale était de transformer le lactose en acide lactique, et dont l'importance pouvait être évaluée par des tests de réduction des colorants comme le bleu de méthylène ou la résazurine (Lück, 1972). À l'inverse, la conservation du lait à basse température entraîne la multiplication des bactéries psychrotrophes (Witter, 1961), qui sont le plus souvent capables de produire des enzymes protéolytiques et lipolytiques (Law, 1979). Ces enzymes sont suffisamment thermostables pour résister aux traitements thermiques habituels du lait, tels que la pasteurisation et le traitement à ultra haute température (UHT) (Mottar, 1981), et ainsi être à l'origine d'une altération des propriétés organoleptiques des produits transformés au cours de leur conservation (Law, 1979; Law et al, 1979; Cousin, 1982; Schröder et al, 1982; Hicks et al, 1986).

L'évaluation rapide de la qualité bactériologique du lait cru, et en particulier du niveau de sa flore psychrotrophe revêt donc un grand intérêt, en particulier pour un tri des laits avant transformation. Or, la méthode de référence pour la numération de la flore psychrotrophe du lait cru (Anonyme, 1981) nécessite une incubation de $10 \mathrm{j}$ à $7{ }^{\circ} \mathrm{C}$, qui est incompatible avec les besoins des entreprises laitières. Dans ce but, des techniques de dénombrement ont été proposées au cours des dernières années afin de réduire la durée d'incubation, comme celle d'Oliveria et Parmelee (1976) ou la technique de dénombrement des Pseudomonas psychrotrophes proposée par Richard et al (1988).

Par ailleurs, une autre voie a été abordée par Hankin et Dillman (1968) qui ont obtenu une bonne relation entre le nombre de colonies cytochrome oxydase positives sur boìte de Petri et le nombre de microorganismes psychrotrophes du lait cru. A la suite de ces travaux, Kroll (1985) a testé la spécificité du dosage de l'activité cytochrome oxydase pour une estimation rapide du nombre de micro-organismes psychrotrophes présents dans le lait cru ou pasteurisé. En effet, d'une façon générale, les bactéries à Gram négatif psychrotrophes possèdent une cytochrome oxydase très active (Steel, 1961), contrairement aux bactéries à Gram négatif non psychrotrophes et aux bactéries à Gram positif (Jurtshuk et McQuitty, 1976a, b; Jurtshuk et Liu, 1983; Kroll, 1985; Kroll et Rodrigues, 1986a).

La cytochrome oxydase est l'enzyme terminale de la chaîne respiratoire qui 
réduit l'oxygène moléculaire en eau. Elle est donc présente dans toutes les cellules aérobies, qui tirent leur énergie du métabolisme de l'oxygène. Le TMPD (NNN'N' tétraméthyl-p-phénylène diamine dihydrochloride) est un substrat spécifique inconstesté de la cytochrome oxydase. II a été utilisé dans de nombreuses études taxonomiques (Kovacs, 1956; Steel, 1961; Stanier et al, 1966) car il apporte rapidement une information qualitative soit pour différencier certaines souches entre elles (Faller et Schleifer, 1981; McEwen et al, 1985), soit pour effectuer un classement des espèces en fonction de leur activité enzymatique (Jurtshuk et McQuitty, 1976a, b; Jurtshuk et Liu, 1983). Des mesures manovolumétriques ont permis d'observer sur des cellules bactériennes de Neisseria catarrhalis et d'Azobacter vinelandii prélevées en fin de phase exponentielle que le TMPD réduit pénètre dans les cellules intactes, où il cède ses électrons à la partie terminale de la chaîne respiratoire (Jurtshuk et Milligan, 1974; Jurtshuk et al, 1975). De plus, l'oxydation du TMPD forme un radical semiquindiimine stable et coloré (bleu-violet), alors que la forme diamine réduite est incolore. Ainsi, l'intensité de la coloration obtenue dans une suspension de bactéries est proportionnelle à l'activité de la cytochrome oxydase globale des cellules aérobies.

Au-delà de $10^{4} \mathrm{UFC} / \mathrm{ml}$, Kroll (1985) a obtenu des coefficients de corrélation de 0,92 et 0,84 entre l'activité cytochrome oxydase et le niveau de la flore psychrotrophe, respectivement sur des laits pasteurisés et des laits crus. Cependant, ces corrélations élevées ont été établies en conditions de laboratoire, c'est-à-dire sur des laits contaminés artificiellement avec des échantillons de lait préalablement incubés pendant des durées relativement longues. De même, Kroll et Rodrigues (1986b) ont testé l'utilisation de ce test pour prédire la durée de conservation des laits pasteurisés. Ils ont obtenu une bonne prédiction dans $89 \%$ des cas, en effectuant le dosage de l'activité cytochrome oxydase après une préincubation des échantillons pendant $18 \mathrm{~h}$ à $20^{\circ} \mathrm{C}$, en présence d'un inhibiteur de la flore à Gram positif.

Dans le cadre de notre étude, nous avons voulu tester l'utilisation d'une mesure de l'activité cytochrome oxydase pour estimer le niveau de la flore psychrotrophe dans les conditions réelles des entreprises laitières, c'est-à-dire sur des échantillons de lait cru prélevés dans les citernes de ramassage à l'arrivée au quai. Toutefois, nous avons modifié la technique de Kroll (1985) en y ajoutant une incubation préliminaire des échantillons destinée à amplifier le signal fourni par la flore psychrotrophe. Nous avons calculé tous les paramètres statistiques de répétabilité et de justesse de la méthode, et en particulier sa précision d'estimation qui n'est pas mentionnée dans l'étude précédente de Kroll (1985). De plus, nous avons comparé deux méthodes de lecture de l'intensité de coloration liée à l'activité cytochrome oxydase : d'une part, une appréciation visuelle par comparaison à une gamme étalon similaire à celle utilisée par Kroll (1985), et d'autre part, une lecture à l'aide d'un colorimètre basé sur la réflectance.

\section{MATÉRIEL ET MÉTHODES}

\section{Origine des échantillons}

Cent vingt échantillons de lait cru de 4 à 6 traites ont été prélevés dans les citernes de ramassage de 4 entreprises laitières françaises, dès leur arrivée au quai de réception; ils ont ensuite été conservés à $0^{\circ} \mathrm{C}$ pendant au plus $24 \mathrm{~h}$ avant analyse (délai imposé par le transport entre les entreprises et le laboratoire). 


\section{Numérations bactériennes}

Avant ensemencement, les échantillons ont été agités pendant $30 \mathrm{~s}$ ả l'aide d'un homogénéisateur à turbine (Ultra-turrax, type 18/10) tournant à $20000 \mathrm{tr} / \mathrm{min}$. Ce mode d'agitation est actuellement celui qui assure la meilleure dispersion des amas microbiens (Richard, 1980; Richard et al, 1981); plus de $95 \%$ des bactéries à Gram négatif se présentent alors sous forme isolée ou d'amas de 2 cellules (Richard, 1980).

Les échantillons ont ensuite été soumis au dénombrement de la flore psychrotrophe sur milieu Plate Count Agar (Difco) après $10 \mathrm{j}$ d'incubation à $7^{\circ} \mathrm{C}$ (Anonyme, 1981).

Les ensemencements ont été réalisés à la surface de la gélose à l'aide de l'appareil Spiral décrit par Gilchrist et al (1973). Nous avons choisi cette méthode car elle est désormais acceptée comme technique de référence (Anonyme, 1985a et 1987). Les analyses ont été effectuées en double, afin d'obtenir une meilleure précision des résultats de numération. Les résultats sont exprimés en unités formant colonies par millilitre de lait (UFC/ml).

\section{Dosage de l'activité cytochrome oxydase}

Un volume de $1 \mathrm{ml}$ d'une solution à $10^{-2} \mathrm{~mol} / \mathrm{l}$ de TMPD (NNN'N' tétraméthyl-p-phénylène diamine dihydrochloride) préparée extemporanément est ajouté à $1 \mathrm{ml}$ de lait réparti dans des cuves pour spectrophotomètre (Kroll, 1985). Après agitation, le mélange réactionnel est laissé à température ambiante $\left(25^{\circ} \mathrm{C}\right)$ pendant 5 $\min ( \pm 5 \mathrm{~s}$ ). II se développe une coloration bleuviolette, dont l'intensité est proportionnelle à l'activité enzymatique de la cytochrome oxydase. Deux modes de lecture ont été utilisés pour évaluer l'intensité de la coloration : une lecture visuelle et une lecture à l'aide d'un colorimètre.

\section{Lecture visuelle de la coloration}

Elle consiste à apprécier l'intensité de coloration à l'œill nu, par comparaison à une gamme éta-
Ion établie expérimentalement. Cette gamme comprend 9 points notés de $0-8$; elle est réalisée à l'aide de concentrations croissantes $(0$; $0,2 ; 0,4 ; 0,6 ; 0,8 ; 1,0 ; 1,5 ; 2,0 ; 6,0 \%)$ d'une solution de violet de gentiane à $0,18 \%$ dans l'éthanol. Ces différentes solutions sont mélangées volume à volume avec du lait UHT (1 ml). II est possible d'effectuer des appréciations au demi point des intensités de coloration.

\section{Lecture de la coloration à l'aide d'un colorimètre}

La colorimétrie utilisée repose sur les propriétés de réflectance des objets éclairés par différents illuminants. Le chromamètre (Minolta CR-200) utilisé dans cette étude, est composé d'une tête de lecture reliée à un minicalculateur. Le système optique de la tête de mesure utilise une illumination diffuse, fournie par une lampe à arc Xénon pulsé (PXA) et contrôlèe par 3 cellules photosensibles. Seule la lumière réfléchie perpendiculairement à la surface de l'échantillon est récupérée par la fibre optique et analysée par 3 autres cellules photosensibles. Ces dernières convertissent la lumière reçue en courant dont l'intensité est proportionnelle à l'éclat de la lumière. Un microprocesseur transforme ensuite ces informations en coordonnées dans l'espace couleur Cielab : la luminance $L^{*}$ et les coordonnées chromatiques $\mathrm{a}^{*}$ et $\mathrm{b}^{*}$ qui correspondent aux valeurs caractéristiques de l'objet sur les 2 axes définis par les couleurs paires antagonistes (vert/rouge pour $\mathrm{a}^{*}$ et jaune/bleu pour $\mathrm{b}^{*}$ ).

Pour utiliser le chromamètre, il suffit d'appliquer la tête de lecture sur l'objet à analyser, et de déclencher le flash lumineux. Les coordonnées $L^{*}, a^{*}$ et $b^{*}$ sont alors affichées sur écran digital; dans le cadre de la présente étude, seule la coordonnée chromatique $b^{*}$ a été utilisée, car elle donne une bonne indication de l'évolution de l'intensité de coloration bleue liée à l'activité cytochrome oxydase. Avant chaque série de mesures, un étalonnage de l'appareil doit être effectué à l'aide d'une plaque de blanc standard, dont les coordonnées dans l'espace couleur sont connues.

L'activité de la cytochrome oxydase a été déterminée en double pour chaque échantillon, après une incubation préliminaire. Trois conditions de préincubation ont été testées : $7 \mathrm{~h}$ d'in- 
cubation à $30^{\circ} \mathrm{C}, 48 \mathrm{~h}$ d'incubation à $4{ }^{\circ} \mathrm{C}$ et $48 \mathrm{~h}$ d'incubation à $4{ }^{\circ} \mathrm{C}$ suivies de $7 \mathrm{~h}$ à $30^{\circ} \mathrm{C}$. La préincubation de $7 \mathrm{~h}$ à $30^{\circ} \mathrm{C}$ résulte d'un compromis entre d'une part, la volonté d'obtenir une information rapide sur la qualité du lait et d'autre part, la nécessité d'amplifier le signal fourni par la flore psychrotrophe. En effet, bien que les échantillons analysés concernaient des laits conservés à basse température pendant 23 j (4-6 traites), ils présentaient rarement à leur arrivée au laboratoire une activité cytochrome oxydase supérieure à 2 en échelle visuelle (sur 80 échantillons analysés, moins de $5 \%$ d'entre eux). Quant à l'incubation de $48 \mathrm{~h}$ à $4^{\circ} \mathrm{C}$, elle a été choisie afin de favoriser la croissance des Pseudomonas du groupe fluorescent qui peuvent être responsables de défauts des qualités organoleptiques du lait et des produits laitiers; à cette température, ce sont les seuls microorganismes capables de se multiplier rapidement dans le lait cru (Richard, 1981a).

\section{Calculs statistiques}

La répétabilité et la justesse des mesures de l'activité cytochrome oxydase ont été calculées conformément aux recommandations de la norme FIL 128 (Anonyme, 1985b). Les calculs statistiques ont été effectués après transformation logarithmique en base 10 des nombres $\mathrm{d}$ 'UFC/ml, afin de normaliser la distribution.

Tous les modes de calcul statistique ont été largement détaillés dans un précédent article (Piton et Rongvaux-Gaïda, 1990); aussi, on ne rappelera ici que les points nécessaires à la compréhension des paramètres statistiques utilisés.

\section{Répétabilité}

L'écart type de répétabilité des mesures de l'activité cytochrome oxydase ( $s_{r}$ en unités d'intensité de coloration) a été établi à partir des 2 mesures effectuées sur chaque échantillon (Anonyme, 1985b). Pour pouvoir comparer la répétabilité de la méthode à celle d'autres techniques microbiologiques, l'écart type de répétabilité est exprimé également en log UFC/ml $\left(s_{r}^{*}\right)$ par l'intermédiaire de la droite de calibrage.
Enfin, on a calculé l'écart type relatif géométrique de répétabilité en données non transiormées, ou GRSD (en \% d'UFC/ml), qui exprime la variabilité des résultats au-dessus de la moyenne géométrique (Piton et Grappin, 1991).

\section{Justesse}

La justesse représente l'étroitesse de l'accord entre la vraie valeur de la grandeur à mesurer et le résultat moyen qui serait obtenu en appliquant la technique expérimentale un grand nombre de fois (Anonyme, 1985b). Dans le cadre de cette étude, l'équation de calibrage $(y=b x+a)$ a été établie conformément à la norme FIL. Le logarithme décimal du nombre d'UFC/ml (valeur vraie) est considéré comme variable dépendante et l'intensité de coloration (moyenne de 2 déterminations obtenues par lecture visuelle ou colorimétrique) comme variable indépendante.

La précision d'estimation de la technique expérimentale est donnée par les limites de l'intervalle statistique de dispersion à l'intérieur duquel se situe, avec une probabilité de $95 \%$, la vraie valeur donnée par la méthode de référence. Si y est le nombre d'UFC/ml calculé à partir de la droite de calibrage, ces limites sont, en valeur logarithmique, $\log y \pm 1,96 \times s_{y, x}$ où $s_{y, x}$ est l'écart type résiduel de la droite de régression. En données non transformées, ces limites sont $y / 10^{1,96 \times s_{y, x}}$ pour la limite inférieure et $y \times$

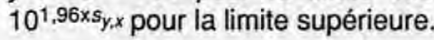

\section{RÉSULTATS}

\section{Comparaison des 2 modes d'estimation de l'intensité de coloration}

La comparaison entre les 2 modes d'estimation de l'intensité de coloration a été effectuée sur l'ensemble des analyses (120 échantillons analysés dans 3 conditions de préincubation différentes). La figure 1 présente la relation existant entre l'appréciation visuelle par comparaison à une 


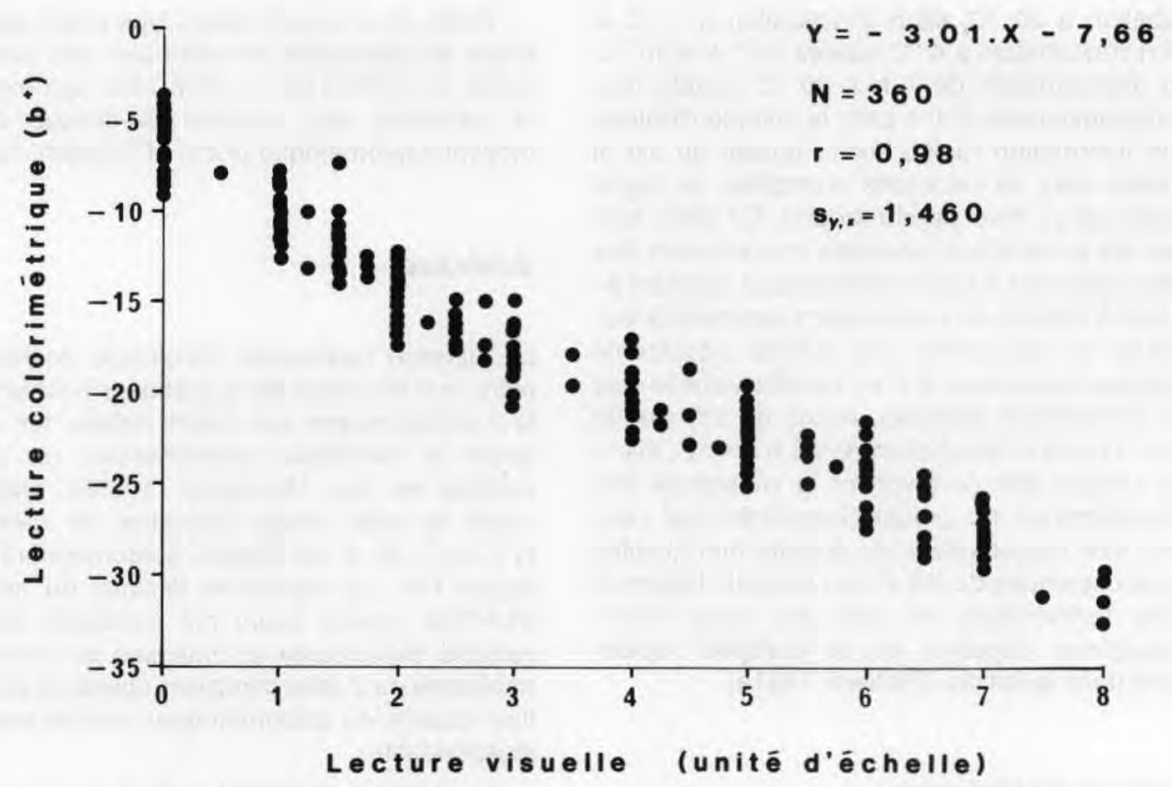

Fig 1. Comparaison entre les mesures de l'intensité de coloration effectuées visuellement (par comparaison à une gamme étalon entre 0 et 8 ) ou à l'aide d'un colorimètre (coordonnée chromatique $b^{*}$ ). Comparison between the measurement of color intensity by visual reading (with a scale from 0 to 8 or by colorimeter (chromatic value $b^{\star}$ ).

gamme étalon et la mesure effectuée au colorimètre. La relation est linéaire et la corrélation obtenue est hautement significative $(r=0,98)$. L'écart type résiduel $\left(s_{y, x}\right)$ est égal à 1,460 (en unité de $\left.b^{\star}\right)$ si on place des résultats de la lecture colorimétrique en variable dépendante, et égal à 0,350 (en point d'échelle) si on place les résultats de la lecture visuelle en variable dépendante. Cela signifie que pour chaque point de l'échelle visuelle, la valeur correspondante de $b^{\star}$ peut varier, dans $95 \%$ des cas, dans un intervalle statistique de dispersion égal à $\pm 1,96 \times 1,460$ soit \pm 2,86 en unité de $b^{\star}$. À l'inverse, pour chaque valeur de $b^{*}$, la valeur sur l'échelle visuelle peut varier dans un intervalle statistique de dispersion égale à $\pm 1,96 \times 0,350$ soit $\pm 0,686$ en point de l'échelle visuelle.

\section{Répétabilité}

Le tableau I présente les écarts types de répétabilité exprimés dans l'unité de lecture et en log d'UFC/ml, ainsi que les écarts types relatifs géométriques pour les 2 techniques de mesure de l'intensité de coloration. La répétabilité des 2 techniques a été calculée sur les 120 échantillons de lait dans chacune des 3 conditions de préincubation. Pour les échantillons analysés après $7 \mathrm{~h}$ d'incubation à $30{ }^{\circ} \mathrm{C}$, nous n'avons pas pu convertir l'écart type de répétabilité en log UFC/ml, car nous n'avons pas calculé la droite de calibrage (cf paragraphe suivant). II n'existe pas de différence significative au seuil de $5 \%$ (test $F$ de comparaison des variances; Dagnelie, 
Tableau I. Répétabilité de la mesure de l'activité cytochrome oxydase déterminée par lecture de l'intensité de coloration visuellement (EV) ou à l'aide d'un colorimètre (Colo).

Repeatability of the cytochrome oxidase activity measure, determined by visual reading of the color intensity (EV) or by colorimeter (Colo).

\begin{tabular}{|c|c|c|c|c|c|c|}
\hline \multirow{2}{*}{$\begin{array}{l}\text { Conditions } \\
\text { de } \\
\text { préincubation }\end{array}$} & \multicolumn{2}{|c|}{$s_{r}$} & \multicolumn{2}{|c|}{$s_{r}^{*}$} & \multicolumn{2}{|c|}{ GRSD } \\
\hline & $E V$ & Colo & $E V$ & Colo & $E V$ & Colo \\
\hline $7 \mathrm{~h}$ à $30^{\circ} \mathrm{C}$ & 0,102 & 0,350 & ND & ND & ND & ND \\
\hline $48 \mathrm{~h}$ à $4^{\circ} \mathrm{C}$ & 0,091 & 0,283 & 0,024 & 0,022 & 5,60 & 5,28 \\
\hline $\begin{array}{l}48 \text { h à } 4^{\circ} \mathrm{C} \\
\text { puis } 7 \text { h à } 30^{\circ} \mathrm{C}\end{array}$ & 0,085 & 0,339 & 0,022 & 0,034 & 5,08 & 8,14 \\
\hline
\end{tabular}

$\mathrm{s}_{\mathrm{r}}$ : écart type de répétabilité exprimé dans l'unité d'origine (en point d'échelle visuelle ou en unités de la coordonnée $\left.b^{*}\right) . s_{r}^{*}$ : écart type de répétabilité exprimé en log UFC/ml par l'intermédiaire de la droite de calibrage. GRSD : écart type relatif géométrique de répétabilité en \% UFC/ml. ND: non déterminé. $s_{r}$. Repeatability standard deviation in reading unit (visual scale or chromatic value $b^{*}$ ); $s_{r}^{*}$ : Repeatability standard deviation in log CFU/ml; GRSD: Repeatability geometric relative standard deviation in \% CFU/ml; ND: Not determined.

1970) entre les variances de répétabilité obtenues pour les 2 méthodes de lecture. Dans tous les cas, l'écart type relatif géométrique de répétabilité en $\% \mathrm{UFC} / \mathrm{ml}$ est faible et nettement inférieur à $10 \%$.

\section{Justesse}

La figure 2 présente les relations obtenues entre le logarithme du nombre de microorganismes psychrotrophes (en UFC/ml) et l'activité enzymatique de la cytochrome oxydase estimée par lecture visuelle de l'intensité de coloration. Pour les échantillons de lait de citerne analysés après $7 \mathrm{~h}$ d'incubation à $30^{\circ} \mathrm{C}$ (fig $2 \mathrm{~A}$ ), il n'existe pas de relation entre le niveau de la flore psychrotrophe initiale et l'activité de la cytochrome oxydase. Par contre, après $48 \mathrm{~h}$ d'incubation à $4{ }^{\circ} \mathrm{C}$ (fig $2 \mathrm{~B}$ ), une relation linéaire entre les 2 variables apparaît nettement, malgré un écart type résiduel assez élevé $\left(s_{y, x}=0,524 \log \mathrm{UFC} / \mathrm{ml}\right)$. Une incubation supplémentaire de $7 \mathrm{~h}$ à $30^{\circ} \mathrm{C}$ n'ap- porte aucune amélioration significative de la précision de la technique (fig $2 \mathrm{C}$ ); on observe seulement une augmentation de l'intensité moyenne de coloration $(1,86$ après $48 \mathrm{~h}$ d'incubation à $4{ }^{\circ} \mathrm{C}$ contre 4,91 avec l'incubation supplémentaire de $7 \mathrm{~h}$ à $\left.30^{\circ} \mathrm{C}\right)$.

La précision d'estimation de l'activité cytochrome oxyase déterminée après $48 \mathrm{~h}$ d'incubation à $4{ }^{\circ} \mathrm{C}$ est donc de $\pm 1,96 \times$ $0,524= \pm 1,027 \mathrm{log} \mathrm{UFC} / \mathrm{ml}$ dans le cas d'une lecture visuelle. Si $y$ est le nombre d'UFC/ml estimé à partir de la droite de calibrage, les limites de l'intervalle statistique de dispersion de $y$ (à $P=0,95$ ) sont en données non transformées, $y / 10^{1,027}$ soit $y / 10,64$ pour la limite inférieure et $y x$ $10^{1,027}$ soit $y \times 10,64$ pour la limite supérieure.

Le tableau II présente les caractéristiques de justesse de la technique après $48 \mathrm{~h}$ d'incubation à $4^{\circ} \mathrm{C}$, dans le cas d'une lecture de l'intensité de coloration au colorimètre. Comme précédemment, il n'existe 


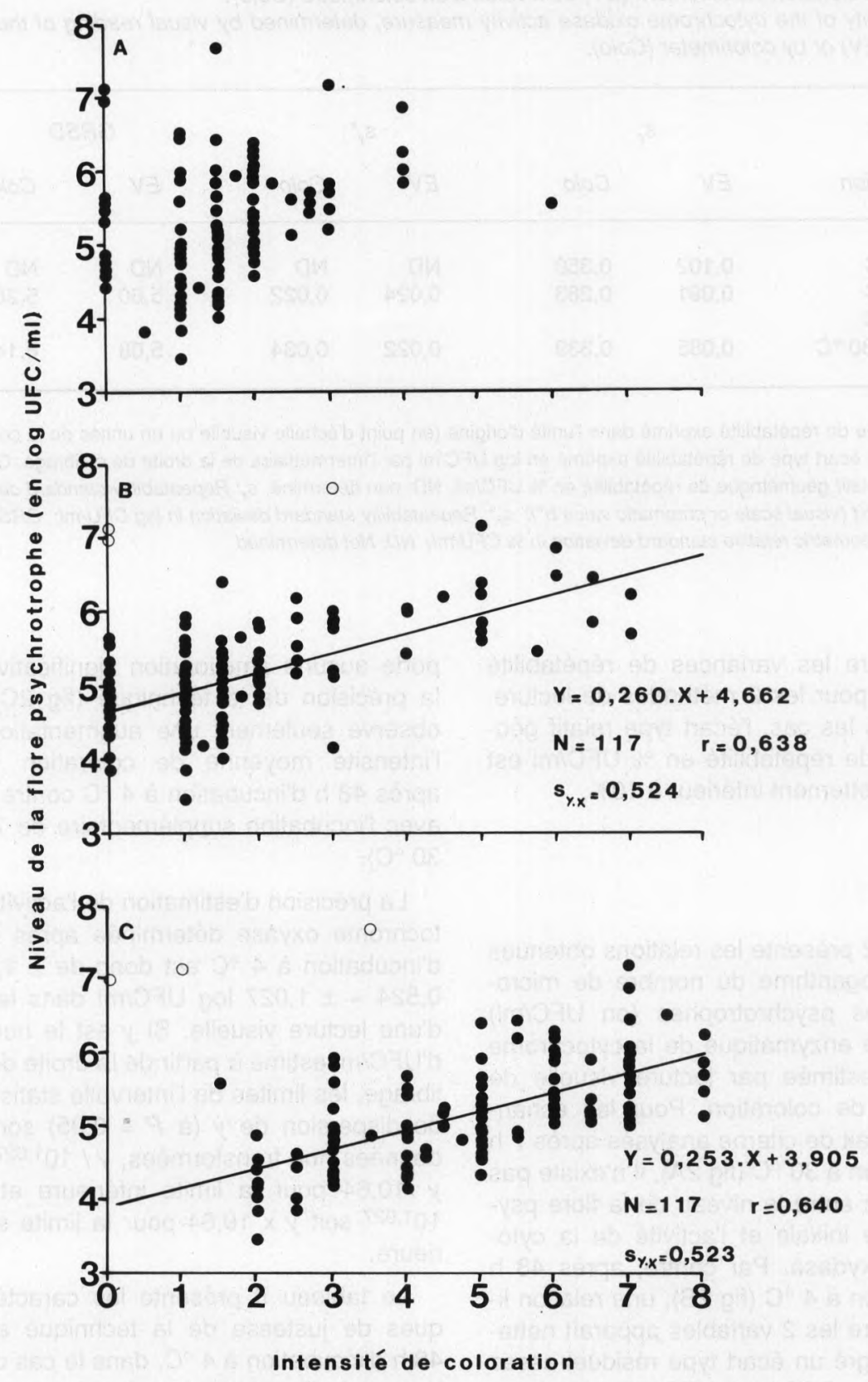


Tableau II. Justesse de la mesure de l'activité cytochrome oxydase déterminée par lecture de l'intensité de coloration à l'aide d'un colorimètre, pour l'estimation du niveau de la flore psychrotrophe du lait cru.

Accuracy of the cytochrome oxidase activity, determined by colorimetric measurement of the color intensity for estimation of the level of psychrotrophic flora in raw milk.

\begin{tabular}{lllll}
\hline $\begin{array}{l}\text { Conditions de } \\
\text { préincubation }\end{array}$ & $\mathrm{N}$ & Equation de calibrage & $\mathrm{s}_{\mathrm{y}, \mathrm{x}}$ & $\mathrm{r}$ \\
\hline $\begin{array}{l}48 \mathrm{~h} \text { à } 4{ }^{\circ} \mathrm{C} \\
48 \mathrm{~h} \text { à } 4{ }^{\circ} \mathrm{C}\end{array}$ & 117 & $\mathrm{y}=-0,079 x+4,116$ & 0,534 & 0,621 \\
puis $7 \mathrm{~h}$ à $30^{\circ} \mathrm{C}$ & 117 & $\mathrm{y}=-0,100 \mathrm{x}+2,864$ & 0,516 & 0,652 \\
\hline
\end{tabular}

$y$ : logarithme du nombre de micro-organismes psychrotrophes/ml (en $\log$ UFC/ml); $x$ : coordonnée chromatique $\mathrm{b}^{*}$; $\mathrm{s}_{\mathrm{y}, \mathrm{x}}$ : écart type résiduel des écarts à la régression (en log UFC/ml); $r$ : coefficient de corrélation; $N$ : nombre d'échantillons inclus dans le calcul de la droite de régression. $y$ : Logarithm of the number of psychrotrophic micro: organisms (in log CFU/ml); $\mathrm{x}$ : Chromatic value $b^{*} ; s_{y, x}$ : Residual standard deviation of the regression (in log CFU) $\mathrm{ml}) ; \mathrm{r}$ : Correlation coefficient; N: Number of samples included in the calculation of the regression line.

pas de différence significative entre les précisions d'estimation obtenues avec ou sans incubation supplémentaire de $7 \mathrm{~h}$ à $30{ }^{\circ} \mathrm{C}$. D'autre part, l'utilisation d'un appareil pour la lecture de l'intensité de coloration n'apporte aucune amélioration de la justesse de la méthode. Après 48 h de préincubation à $4^{\circ} \mathrm{C}$ par exemple, l'écart type résiduel de la régression est de 0,524 log $\mathrm{UFC} / \mathrm{ml}$ dans le cas d'une lecture visuelle, contre 0,534 log UFC/ml dans le cas d'une lecture au colorimètre.

\section{DISCUSSION}

Contrairement aux conclusions émises par Kroll (1985), le dosage de l'activité cytochrome oxydase sur des échantillons de lait cru prélevés en conditions réelles (citernes de ramassage) ne permet pas une évaluation satisfaisante de la flore psychrotrophe, sauf après une incubation préliminaire des échantillons pendant $48 \mathrm{~h}$ à $4^{\circ} \mathrm{C}$. La différence entre les conclusions de ces 2 études peut trouver une explica-

Fig 2. Relation entre le logarithme du nombre initial de micro-organismes psychrotrophes et l'activité cytochrome oxydase mesurée par lecture visuelle de l'intensité de coloration. A. analyse après $7 \mathrm{~h}$ d'incubation à $30^{\circ} \mathrm{C}$. B. analyse après $48 \mathrm{~h}$ d'incubation à $4^{\circ} \mathrm{C}$. C. analyse après $48 \mathrm{~h}$ d'incubation à $4^{\circ} \mathrm{C}$, puis de $7 \mathrm{~h}$ à $30^{\circ} \mathrm{C}$. Les droites de régression ont été établies sur l'ensemble des échantillons, après élimination de 3 d'entre eux (0) qui s'écartaient de plus de 3 écarts types résiduels de ces droites.

Relationship between the initial number of psychrotrophic bacteria (in log CFU/ml) and the cytochrome oxidase enzymatic activity determined by visual reading of the color intensity. A. Measurement after incubation of the milk samples for $7 \mathrm{~h}$ at $30^{\circ} \mathrm{C}$. B. Measurement after incubation of the milk samples at $4{ }^{\circ} \mathrm{C}$ for $48 \mathrm{~h}$. C. Measurement after incubation of the milk samples for $48 \mathrm{~h}$ at $4{ }^{\circ} \mathrm{C}$ and then for $7 \mathrm{~h}$ at $30^{\circ} \mathrm{C}$. The regression lines have been calculated on all the samples after elimination of 3 of them (o) which were 3 residual standard deviations apart from these lines. 
tion au niveau de la nature des échantillons analysés. En effet, Kroll (1985) a utilisé des échantillons ensemencés avec des laits pasteurisés incubés pendant $7 \mathrm{j}$ à basse température. De plus, il a analysé ces échantillons à des intervalles réguliers au cours de leur conservation prolongée (7j) à 4 et $7{ }^{\circ} \mathrm{C}$. Ces conditions opératoires ont inévitablement induit la sélection d'une flore psychrotrophe, en phase exponentielle de croissance, qui est vraisemblablement peu représentative de celle des laits des citernes de ramassage à l'arrivée au quai.

Même si Kroll (1985) n'a pas calculé la précision d'estimation de la technique, on peut noter, dans son étude, une dispersion d'environ $\pm 1,0 \mathrm{log}$ UFC/ml autour de la droite de régression, qui est sensiblement identique à celle que nous avons obtenue sur nos échantillons après $48 \mathrm{~h}$ d'incubation à $4^{\circ} \mathrm{C}$. Cette dispersion assez grande autour de la droite de régression s'explique en particulier par des variations de la nature de la flore psychrotrophe. Par exemple, certaines souches de Pseudomonas psychrotrophes, possèdent une cytochrome oxydase active mais sont déficientes au niveau du cytochrome c, qui assure le transfert des électrons entre le substrat et l'enzyme (Stanier et al, 1966; Richard, 1981b). De plus, l'activité enzymatique de la cytochrome oxydase au sein d'une même cellule dépend de son état physiologique, de son métabolisme, ainsi que de la phase de croissance au moment de l'analyse (Jurtshuk et McQuitty, 1976b).

Après seulement $7 \mathrm{~h}$ d'incubation à $30^{\circ} \mathrm{C}$, l'absence de relation entre l'activité cytochrome oxydase et le niveau de la flore psychrotrophe initiale peut sans doute s'expliquer par le choix de la température de préincubation. En effet, il est vraisemblable que l'incubation à cette température ait modifié les proportions des flores présentes dans l'échantillon. En par- ticulier, elle a pu permettre le développement de souches de micro-organismes non psychrotrophes à cytochrome oxydase positive.

En conclusion, le dosage de l'activité cytochrome oxydase du lait cru aurait pu se révéler comme un test de screening intéressant à l'arrivée au quai à l'usine, compte tenu de son faible coût, de sa simplicité de réalisation, de sa fidélité et des possibilités d'automatisation de lecture qu'offrait l'utilisation d'un colorimètre. $\mathrm{Ce}$ pendant, avec une précision d'estimation d'environ $\pm 1 \mathrm{log}$ UFC/ml, la justesse de ce dosage pour l'évaluation de la flore psychrotrophe est assez médiocre et surtout, la préincubation de $48 \mathrm{~h}$ limite très fortement son intérêt pour l'industriel. Toutefois, il serait intéressant d'étudier la possibilité de réduire cette durée de préincubation, en augmentant la température d'incubation, à $7^{\circ} \mathrm{C}$ par exemple.

Enfin, sur un plan beaucoup plus général, cette étude démontre nettement l'intérêt d'évaluer une technique analytique sur une population issue des échantillons habituellement analysés par les entreprises laitières. En effet, l'emploi d'échantillons contaminés avec des souches pures ou de laits maturés peut conduire à une amélioration artificielle des caractéristiques analytiques des techniques étudiées et à l'utilisation inadaptée de certaines d'entre elles.

\section{REMERCIEMENTS}

Les auteurs remercient MM Lenoir et Auberger (INA Paris-Grignon, laboratoire de la chaire de technologie, route de Thiverval, 78850 ThivervalGrignon) pour leur collaboration lors de la mise en cuvre de la technique de dosage de l'activité cytochrome oxydase du lait cru.

Les auteurs tiennent également à remercier les entreprises laitières pour le prélèvement et l'envoi des échantillons de lait : 
- la société Bel : Centre de recherches, 21 avenue de Ronsard, 41100 Vendôme;

- la société Gervais-Danone (BSN) par l'intermédiaire de la société Stenval : 5 route de Blanche, 59270 Bailleul;

- la société Yoplait : l'usine d'Amiens (Sully, 80000 ) et l'usine de Ressons/Matz (Elnor, 60490).

Leurs remerciements s'adressent également à $M$ Janin (INRA, centre de recherches de Nancy, station de recherches sur la qualité des bois, Champenoux, 54280 Seichamps) pour le prêt du chromamètre.

Ce travail a reçu le soutien financier de la $\mathrm{Di}$ rection générale de l'alimentation du ministère de l'Agriculture (Contrat DGAL nR88/11).

\section{RÉFÉRENCES}

Anonyme (1981) Liquid milk. Enumeration of psychrotrophic microorganisms. Colony count at $6.5^{\circ} \mathrm{C}$. IDF FIL provisional standard 101

Anonyme (1985a) Standard methods for the examination of dairy products. 15th edn, Am Public Health Assoc, New York, USA

Anonyme (1985b) Milk. Definition and evaluation of the overall accuracy of indirect methods of milk analysis. Application to calibration procedure and quality control in dairy laboratory. IDF-FIL provisional standard 128

Anonyme (1987) Microbiologie alimentaire. Ensemencement et dénombrement des microorganismes à l'aide du système «Spiral». Norme française NF V 08-100 AFNOR, Paris

Cousin MA (1982) Presence and activity of psychrotrophic microorganisms in milk and dairy products: a review. J Food Prot 45, 172-207

Dagnelie P (1970) Théorie et méthodes statistiques. Vol 2. Les méthodes de l'inférence statistique. Duculot SA, Gembloux

Faller A, Schleifer KH (1981) Modified oxidase and benzidine tests for separation of staphylococci from micrococci. J Clin Microbiol 6, 1031-1035

Gilchrist JE, Campbell JE, Donnelly CB, Peeler JT, Delaney JM (1973) Spiral plate method for bacterial determination. App/ Microbiol 25, 244-252
Hankin L, Dillman WF (1968) A rapid test to find "potentially" psychrotrophic organisms in pasteurized dairy products. J Milk Food Technol 31, 141-145

Hicks CL, Onuorah C, O'Leary J, Langlois BE (1986) Effect of milk quality and low temperature storage on cheese yield. A summation. J Dairy Sci 69, 649-657

Jurtshuk PJR, Milligan TW (1974) Preliminary characterization studies on the Neisseria catarrhalis electron transport chain. J Bacteriol 120, 552-555

Jurtshuk PJR, McQuitty DN (1976a) Survey of oxidase-positive and negative bacteria using a quantitative Kovacs oxidase test. Int $J$ Syst Bacteriol 26, 127-135

Jurtshuk PJR, McQuitty DN (1976b) Use of a quantitative oxidase test for characterizing oxidative metabolism in bacteria. Appl Environ Microbiol 31, 668-679

Jurtshuk PJR, Liu JK (1983) Cytochrome oxidase analyses of Bacillus strains: existence of oxidase-positive species. Int J Syst Bacteriol 33, 887-891

Jurtshuk PJR, Marcucci OM, McQuitty DN (1975) Tetramethyl- $\rho$-phenylenediamine oxidase reaction in Azobacter vinelandii. Appl Microbio/ 30, 951-958

Kovacs N (1956) Identification of Pseudomonas pyocyanea by the oxidase reaction. Nature (London) 178, 703-703

Kroll RG (1985) The cytochrome $c$ oxidase test for the rapid detection of psychrotrophic bacteria in milk. J Appl Bacteriol 59, 137-141

Kroll RG, Rodrigues UM (1986a) The direct epifluorescent filter technique, cytochrome $c$ oxidase test and plate count method for predicting the keeping quality of pasteurized cream. Food Microbiol 3, 185-194

Kroll RG, Rodrigues UM (1986b) Prediction of the keeping quality of pasteurized milk by the detection of cytochrome $c$ oxidase. J Appl Bacteriol 60, 21-27

Law BA (1979) Reviews of the progress of dairy science: enzymes of psychrotrophic bacteria and their effects on milk and milk products. $\checkmark$ Dairy Res 46, 573-588

Law BA, Andrews AT, Cliffe AJ, Sharpe ME, Chapman HR (1979) Effect of proteolytic raw milk psychrotrophs on Cheddar cheese- 
making with stored milk. J Dairy Res 46, 497-509

Lück H (1972) Bacteriological quality tests for bulk-cooled milk. Dairy Sci Abstr 34, 101-122

McEwen JE, Cameron VL, Poyton RO (1985) Rapid method for isolation and screening of cytochrome $c$ oxidase-deficient mutants of Saccharomyces cerevisiae. J Bacteriol 161, 831-835

Mottar J (1981) Heat resistant enzymes in UHT milk and their influence on sensory changes during uncooled storage. Milchwissenschaft 36, 87-91

Oliveria JS, Parmelee CE (1976) Rapid enumeration of psychrotrophic bacteria in raw milk and pasteurized milk. J Milk Food Technol 39, 269-272

Piton C, Rongvaux-Gaïda D (1990) Estimation par conductimétrie de la flore aérobie mésophile, des bactéries coliformes et de la flore psychrotrophe du lait cru. Lait 70, 293-306

Piton C, Grappin R (1991) A model for statistical evaluation of precision parameters of microbiological methods: application to the dry rehydratable film methods and the IDF reference methods for the enumeration of total aerobic mesophilic flora and coliforms in raw milk. J Assoc Off Anal Chem 74, 92-103

Richard J (1980) Influence de l'agitation du lait cru sur les résultats de dénombrement de sa flore totale à l'aide d'une anse calibrée. Lait $60,211-225$
Richard J (1981a) Influence de diverses méthodes de nettoyage des machines à traire sur la "qualité de conservation" du lait cru à basse température. Lait 61, 354-369

Richard J (1981b) Classification adansonnienne et identification des Pseudomonas psychrotrophes isolés du lait cru conservé à basse température. Ann Microbiol 132a, 171-182

Richard J, Krebs C, Walter J, Grappin R (1981) Comparaison de divers modes d'agitation des échantillons de lait cru en vue de la numération de sa flore totale suivant la méthode Thompson. Lait 61, 250-260

Richard J, Piton C, Gratadoux JJ (1988) Nature des colonies se développant à la surface du milieu Violet Rouge Bile Lactose Agar (VRB) ensemencé avec du lait cru. Validité des dénombrements des colonies lactose-positives et lactose-négatives se développant sur ce milieu. Lait 68, 311-322

Schröder MJA, Cousins CM, McKinnon $\mathrm{CH}$ (1982) Effects of psychrotrophic postpasteurization contamination on the keeping quality at 11 and $5{ }^{\circ} \mathrm{C}$ of HTST-pasteurized milk in the UK. J Dairy Sci 49, 619-630

Stanier RY, Palleroni NJ, Doudoroff M (1966) The aerobic Pseudomonas: a taxonomic study. J Gen Microbiol 43, 159-271

Steel KJ (1961) The oxidase reaction as a taxonomic tool. J Gen Microbiol 25, 297-306

Witter LD (1961) Psychrotrophic bacteria. A review. J Dairy Sci 44, 983-1015 